\title{
INTERAÇÕES MEDICAMENTOSAS ENVOLVENDO CARBONATO DE LÍTIO EM PRESCRIÇÕES DE PACIENTES DE UMA CLÍNICA DE REABILITAÇÃO DE CURITIBA - PR
}

\author{
DRUG-DRUG INTERACTIONS OF LITHIUM CARBONATE IN PRESCRIPTIONS OF \\ PATIENTS IN A CLINIC OF CURITIBA - PR
}

\author{
Roxanne Reis RASDAL ${ }^{1}$; Susan Amaral JAIGOBIND'; Cristiane da Silva PAULA ${ }^{2}$
}

1 - Acadêmica do curso de Farmácia - Universidade Federal do Paraná - UFPR, Curitiba, PR, Brasil.

2 - Docente do curso de Farmácia - Universidade Federal do Paraná - UFPR, Curitiba, PR, Brasil.

Autor para correspondência: crisspaula@onda.com.br

\section{RESUMO:}

O carbonato de lítio é medicamento estabilizador de humor utilizado no tratamento do transtorno bipolar que acomete cerca de $1 \%$ da população brasileira, sendo a patologia mais associada ao uso indevido de substâncias psicoativas que levam à dependência química. O tratamento desta condição é complexo e pode utilizar a polimedicação como ferramenta farmacoterapêutica, o que dá margem à possibilidade da ocorrência de interações medicamentosas. Este trabalho teve o objetivo de analisar prescrições médicas contendo carbonato de lítio e identificar a presença de interações medicamentosas, em uma clínica de reabilitação. Foram analisadas prescrições de cinco pacientes no período de três semanas de terapia, utilizando como fonte de informação as plataformas Drugs.com com acesso livre na internet. Foram localizadas nas prescrições analisadas 19 interações medicamentosas envolvendo carbonato de lítio. Na maior parte dos casos a conduta clínica recomendada é o monitoramento do paciente com relação à intoxicação pelo lítio e ao aparecimento de reações adversas. Fica evidente a necessidade do profissional farmacêutico na equipe multiprofissional, prestando cuidado ao paciente, de forma a otimizar a farmacoterapia, promover saúde e bem-estar e prevenir doenças.

Palavras-chave: Carbonato de lítio; Interações medicamentosas; Prescrições.

\section{ABSTRACT:}

Lithium carbonate is a mood stabilizing drug used in the treatment of bipolar disorder that affects about $1 \%$ of the Brazilian population, and the pathology is most associated with the undue use of psychoactive substances that lead to chemical dependence. The treatment of this condition is complex and can use the polymedication as a pharmacotherapeutic tool, which gives to the possibility of the occurrence of drug interactions. This study aimed to analyze medical prescriptions containing lithium carbonate and to identify the presence of drug interactions in a rehabilitation clinic. Prescriptions of five patients were analyzed during three weeks of therapy using Drugs.com platforms with free access on the internet. A total of 19 drug interactions involving lithium carbonate were found in the prescriptions. In most cases, the recommended clinical management is the monitoring of the patient in relation to Lithium toxicity and the appearance of adverse reactions. It is evident the need of the pharmaceutical professional in the multiprofessional team, giving care to the patient, in order to optimize pharmacotherapy, promote health and well-being and prevent diseases.

Keywords: Lithium carbonate; Drug interactions; Prescriptions. 


\section{INTRODUÇÃO}

O uso do lítio como agente terapêutico teve início em 1859 no tratamento da gota por suas propriedades alcalinas (HEALY, 2008) e seu efeito psicotrópico foi descoberto em 1949 por Cade que observou melhora rápida em um grupo de pacientes com mania. (RANG, 2013).

É um dos agentes antimaníacos mais prescrito para tratar e prevenir o transtorno bipolar (TB) (GOLDBERG, 2000; GOODWIN, ZIS, 1979). Não apresenta ação sedativa, depressora ou euforizante e seu mecanismo de ação na mania ainda não foi esclarecido, apesar de saber que é capaz de alterar o transporte de sódio em células nervosas e musculares e efetua uma mudança no metabolismo intraneuronal das catecolaminas. (DYNAMED, 2016).

O lítio $\left(\mathrm{Li}^{+}\right)$é a terapêutica em longo prazo mais eficiente no tratamento e prevenção dos TBs (TISSINI, 2013) como estabilizador do humor. Quando em uso, é necessário que haja monitoramento constante da concentração plasmática para evitar casos de intoxicações, pois se trata de substância de baixo índice terapêutico, além de potencial problema no caso de pacientes com lesão renal e de interações medicamentosas. (DYNAMED, 2016).

O TB acomete cerca de $1 \%$ da população brasileira, sendo a patologia muito relacionada ao uso indevido de substâncias psicoativas que levam à dependência química (HANEMANN, 2010; PULCHERIO et al., 2008; RIBEIRO et al., 2005). A combinação dependência química e patologia psiquiátrica, gera uma condição complexa que leva a necessidade de uso por parte do paciente, da associação de vários fármacos como ferramenta terapêutica. Quando dois ou mais medicamentos são utilizados concomitantemente, eles podem produzir resultados de forma independente ou interagir entre si, promovendo aumento ou redução dos efeitos terapêuticos inicialmente esperados, ou até mesmo promover toxicidade. Diversos mecanismos de interações entre medicamentos podem afetar aspectos biofarmacêuticos, farmacocinéticos ou farmacodinâmicos, alterando o resultado esperado da farmacoterapia. (CORRER, OTUKI, 2013).

Nesta perspectiva, este estudo teve por objetivo realizar um levantamento das prescrições médicas, que chegam à farmácia de uma Clínica de Reabilitação, que contenham Carbonato de Lítio associado a um ou mais medicamentos, analisar possíveis interações do fármaco com os demais medicamentos prescritos e verificar qual a conduta 
adequada para cada caso.

\section{METODOLOGIA}

Trata-se de um estudo retrospectivo e descritivo, desenvolvido no setor de farmácia de uma clínica de reabilitação que atende pacientes com distúrbios psiquiátricos e dependência química, localizada na cidade de Curitiba - PR. Foi realizada análise das prescrições médicas dos pacientes internados que faziam uso do carbonato de lítio associado a outros medicamentos na data da coleta das informações. As interações medicamentosas entre carbonato de lítio e os outros medicamentos foram listadas, e as informações sobre sua gravidade e conduta clínica recomendada foram relacionadas. Nestes mesmos pacientes foram identificadas interações medicamentosas entre os outros medicamentos que não o carbonato de lítio. Para cada prescrição de paciente selecionada foram analisadas prescrições de até três semanas anteriores ao dia da coleta, para buscar informações sobre possíveis medicamentos utilizados anteriormente e suspensos. Para avaliar as potenciais interações presentes nas prescrições e conduta clínica recomendada, foram utilizadas recomendações descritas na plataforma Drugs.com (https://www.drugs.com /drug_interactions.php) de livre acesso. As interações foram classificadas em "graves", "moderadas" e "leves", que são definidas da seguinte maneira: "graves" - podem oferecer risco de morte e/ou requerer intervenção médica urgente para minimizar efeitos adversos graves; "moderadas" - pode resultar em exacerbação das condições clínicas do paciente e/ou requerer troca de terapia; "menores" (leves) - interações medicamentosas com efeitos clínicos limitados, podendo sua manifestação incluir aumento da frequência ou severidade dos efeitos colaterais, mas não requerem alterações importantes na terapia.

O protocolo de pesquisa cumpriu os princípios éticos contidos na Declaração de Helsinki e as normas da resolução n 196/96 do Conselho Nacional de Saúde, tendo sido aprovado pelo Comitê de Ética em Pesquisa do Setor de Ciências da Saúde da

Universidade Federal do Paraná, sob o número CEP/SD 1.714 .941 e CAAE: 57660316.5.0000.0102.

\section{RESULTADOS E DISCUSSÃO}

A data da análise foi no dia 15 de setembro do ano de 2016. Havia na farmácia 41 prescrições de pacientes hospitalizados e, destas, cinco apresentavam carbonato de lítio 
associado a outros medicamentos. A faixa etária dos pacientes variou de 20 a 42 anos e quatro eram do sexo masculino, com diagnósticos de depressão e históricos de dependência química ao álcool, maconha, cocaína, LSD ou tabaco.

Levando em consideração as três semanas anteriores à data da coleta das informações, estes pacientes possuíam prescrições diárias, e no período avaliado houve alterações das mesmas através de ajustes posológicos, inclusão ou exclusão de fármacos, dependendo da necessidade de cada um após avaliação médica. Observou-se 19 medicamentos diferentes com possibilidade de interação medicamentosa com o carbonato de lítio como ilustrado na Tabela 1. Não foi encontrada nenhuma contraindicação.

TABELA 1 - POSSIBILIDADE DE INTERAÇÕES MEDICAMENTOSA NAS PRESCRIÇÕES MÉDICAS DA CLÍNICA DE REABILITAÇÃO, FÁRMACOS ENVOLVIDOS, FREQUÊNCIA E CLASSIFICAÇÃO

\begin{tabular}{|c|c|c|c|}
\hline CLASSIFICAÇĀO & CARBONATO DE & CLASSE DO MEDICAMENTO & FREQUËNCIA** (\%) \\
\hline DA INTERAÇÃO* & LÍTIO ASSOCIADO À: & & \\
\hline \multirow[t]{6}{*}{ GRAVE } & Citalopram & Antidepressivo ISRS & 20 \\
\hline & Duloxetina & Antidepressivo IRSN & 20 \\
\hline & Escitalopram & Antidepressivo ISRS & 20 \\
\hline & Haloperidol & Antipsicóticos & 20 \\
\hline & Sertralina & Antidepressivo ISRS & 40 \\
\hline & Trazodona & Antidepressivo ISRS & 20 \\
\hline \multirow[t]{11}{*}{ MODERADA } & Ácido valpróico & Estabilizador de humor & 20 \\
\hline & Clonazepam & Benzodiazepínico/ ansiolítico & 60 \\
\hline & Divalproato de sódio & Estabilizador de humor & 60 \\
\hline & Eritromicina & Antibiótico & 20 \\
\hline & Mirtazapina & Antidepressivo tetracíclico & 20 \\
\hline & Oxicodona & Analgésico opioide & 20 \\
\hline & Prometazina & Anti-histamínico & 20 \\
\hline & Quetiapina & Antipsicóticos & 80 \\
\hline & Risperidona & Antipsicóticos & 20 \\
\hline & Topiramato & $\begin{array}{l}\text { Anticonvulsivante, estabilizador } \\
\text { do humor }\end{array}$ & 20 \\
\hline & Zolpidem & Hipnótico & 60 \\
\hline \multirow[t]{2}{*}{ LEVE } & Diazepam & Benzodiazepínico/ ansiolítico & 20 \\
\hline & Lorazepam & Benzodiazepínico/ ansiolítico & 20 \\
\hline
\end{tabular}

* plataforma Drugs.com (2016). ${ }^{\star *} 100 \%$ = encontrada nas 5 prescrições; $80 \%$ = encontrada em 4 prescrições; $60 \%=$ encontrada em 3 prescrições; $40 \%=$ encontrada em 2 prescrições; $20 \%=$ em 1 prescrição. IRSN = inibidor da recaptação de serotonina e noradrenalina; ISRS = inibidor seletivo da recaptação de serotonina.

FONTE: As autoras (2016). 
As interações consideradas Graves envolvem o uso do carbonato de lítio com antidepressivos ISRS, IRSN e agente antipsicótico (RANG, 2013). Os fármacos ISRS e os IRSN promovem aumento da serotonina na fenda sináptica. O lítio pode aumentar os efeitos farmacológicos dos ISRS e IRSN, e potencializar o risco de síndrome serotoninérgica, que é uma condição rara, porém grave e potencialmente fatal. Acredita-se que ocorra como resultado da hiperestimulação dos receptores 5-HT1A e $2 \mathrm{~A}$ do tronco encefálico. $\mathrm{O}$ mecanismo exato pelo qual o lítio aumenta a atividade serotonérgica é desconhecido, porém, os sintomas podem incluir alterações do estado mental (irritabilidade, consciência alterada, confusão, alucinações e coma), disfunção autonômica (taquicardia, hipertermia, diaforese, tremores, labilidade da pressão arterial e midríase), anormalidades neuromusculares (hiperreflexia, mioclonia, tremor, rigidez e ataxia) e sintomas gastrointestinais (cólicas abdominais, náuseas, vômitos e diarreia) (DRUGS.COM, 2016, DYNAMED, 2016, MEDSCAPE, 2016). Recomenda-se precaução se o lítio for prescrito em combinação com ISRS ou IRSN. Os níveis de lítio devem ser avaliados regularmente e a dose ajustada se necessário. Os pacientes devem ser monitorados (DRUGS.COM, 2016; MEDSCAPE, 2016) e se houver suspeita ou confirmação da síndrome todos os fármacos serotoninérgicos devem ser interrompidos imediatamente e os cuidados de suporte devem ser prestados conforme necessário. (DRUGS.COM, 2016).

Com relação ao haloperidol também foi observado que o mesmo pode causar prolongamento do intervalo QT dependente da dose, e quando associado ao lítio pode resultar em efeitos aditivos com aumento do risco de arritmias ventriculares, como torsade de pointes e morte súbita (DRUGS.COM, 2016). O intervalo QT é um parâmetro eletrocardiográfico que representa a duração da sístole, e valores altos estão associados com arritmias com sintomas que incluem tontura, torsades de pointes (taquicardia ventricular polimórfica) e em alguns casos fibrilação ventricular e morte súbita (OLIVEIRA JUNIOR et al., 2004). O aumento do intervalo QT não é uma condição frequente, e desta forma não é possível prever sua ocorrência. Sabe-se que pacientes que possuem fatores de risco como doença cardíaca, distúrbios eletrolíticos ou síndrome do QT longo congênito podem ser mais propensos. Os pacientes devem ser aconselhados a procurar o médico se sentirem sintomas sugestivos de torsade de pointes, como tontura, falta de ar, palpitação e desmaios. (DRUGS.COM, 2016).

Este efeito também é observado quando o lítio é associado ao citalopram. A literatura recomenda que quando a associação é necessária, o citalopram deve ser administrado em doses abaixo de $40 \mathrm{mg} / \mathrm{dia}$ (DRUGS.COM, 2016). Com relação ao 
haloperidol, embora tenha sido usado associado ao lítio com segurança em muitos pacientes, houve registros de casos de síndrome encefalopática consistindo de graves efeitos neurotóxicos (DRUGS.COM, 2016; MEDSCAPE, 2016; DYNAMED, 2016) e sintomas extrapiramidais, seguidos de danos cerebrais irreversíveis, e neste caso recomenda-se precaução particularmente quando administrado por via intravenosa ou em doses superiores às recomendadas. (DRUGS.COM, 2016).

As interações consideradas moderadas envolvem fármacos antipsicóticos (quetiapina, risperidona), estabilizadores do humor (ácido valpróico, divalproato de sódio, topiramato), benzodiazepínico (clonazepam), antidepressivo tetracíclico (mirtazapina), hipnótico (zolpidem), anti-histamínico (prometazina), analgésico opióide (oxicodona) e antibiótico (eritromicina).

Com relação à associação de lítio com quetiapina a literatura recomenda que deve ser evitada tendo em vista a possibilidade de efeitos cardiovasculares aditivos em combinação com outros fármacos que também prolongam o intervalo QT. Recomenda-se precaução e monitorização clínica para identificar a presença de torsade de pointes, tais como tonturas, tonturas, desmaios, palpitação, ritmo cardíaco irregular, falta de ar ou síncope, se for necessário o uso concomitante (DRUGS.COM, 2016). Apesar desta recomendação existem autores que não consideram existir interação medicamentosa entre estes dois fármacos. (MEDSCAPE, 2016).

A coadministração de lítio com risperidona pode estar associada a casos raros de síndrome encefalopática (fraqueza, letargia, febre, tremores, confusão, sintomas extrapiramidais, leucocitose e elevação das enzimas hepáticas e nitrogênio ureico no sangue) sendo recomendada monitorização rigorosa dos efeitos adversos. Podem ser necessários ajustes posológicos ou descontinuação de um ou ambos os fármacos se for suspeitada uma interação (DRUGS.COM, 2016). Boora et al. (2008) relataram um caso em que um paciente desenvolveu encefalopatia poucos dias após o início da associação, com reversão do quadro após a descontinuação da farmacoterapia. Vacheron-Trystram et al. (2004) acreditam que a risperidona seja uma boa opção combinada ao carbonato de lítio, e que os efeitos adversos encontrados foram poucos, demonstrando que a associação é segura.

De acordo com Ketter (2008), o FDA aprova a combinação de medicamentos para o tratamento de estados maníacos agudos, depressão bipolar aguda e terapia de manutenção, sendo que nesse último o recomendado é utilizar quetiapina juntamente com carbonato de lítio ou valproato de sódio. Entretanto, médicos ao prescrever tais associações 
enfrentam desafios, pois apesar de ser benéfico, o sinergismo de efeitos farmacológicos, cada medicamento possui mecanismos de ação, efeitos adversos e efeitos terapêuticos diferentes. Dessa maneira, a decisão de experimentar uma terapia combinada reside em avaliar a tolerabilidade, a eficácia e a segurança dos medicamentos envolvidos, bem como a resposta individual de cada paciente. (KETTER et al., 2008).

A associação de lítio com ácido valpróico, valproato de sódio, clonazepam, mirtazapina, zolpidem e oxicodona requer monitoramento quanto a efeitos depressivos do Sistema Nervoso Central e depressão respiratória. Pode ser necessária uma titulação cuidadosa da dosagem, particularmente no início do tratamento. Os pacientes ambulatoriais devem ser aconselhados a evitar atividades perigosas que exijam atenção mental e coordenação motora. (DRUGS.COM, 2016).

A coadministração com topiramato pode aumentar as concentrações séricas de lítio por mecanismo não conhecido, sendo recomendada precaução na associação. Os pacientes devem ser aconselhados a informar ao médico caso experimentem sintomas de toxicidade ao lítio, tais como sonolência, tonturas, diarreia, vômitos, fraqueza muscular, ataxia, tremor, visão turva, zumbido, sede excessiva e aumento da micção. (DRUGS.COM, 2016).

Com relação à prometazina, é comum na terapêutica da mania aguda a associação com lítio, porém uma série de efeitos neurológicos e psiquiátricos pode ocorrer tais como alterações no estado mental, delírio, convulsões, efeitos extrapiramidais, febre e outros sintomas foram relatados. A recomendação é de que os pacientes que recebem lítio e uma fenotiazina sejam cuidadosamente monitorados quanto à eficácia e segurança e em alguns casos, a interrupção de um ou ambos os agentes pode ser necessária. (DRUGS.COM, 2016).

Teoricamente, o uso concomitante de lítio e eritromicina, dois medicamentos que podem causar prolongamento do intervalo QT, pode levar a efeitos aditivos e aumento do risco de arritmias ventriculares, incluindo torsade de pointers e morte súbita. Entretanto, no caso da prescrição analisada, este fato deve ser desconsiderado, tendo em vista que o paciente faz uso tópico do antibiótico, e para esta forma farmacêutica, não existe informação descrita na literatura. (DRUGS.COM, 2016).

Foram encontradas duas interações consideradas leve, que são de significado clínico mínimo, e na maior parte dos casos não requer nenhuma alteração na farmacoterapia. Foi notificada hipotermia em um paciente que associou diazepam e lítio, mas não é conhecido se outros benzodiazepínicos podem promover o mesmo sintoma pela 
interação, como por exemplo o lorazepam que constava em uma das prescrições (DRUGS.COM, 2016). Similarmente em seu trabalho, Viel et al. (2014) ao avaliar a ocorrência de potenciais interações medicamentosas com os benzodiazepínicos encontrou o lítio em possíveis interações classificadas com leves.

Apesar de terem sido encontradas interações medicamentosas nas prescrições analisadas neste estudo, é necessário discernir a teoria da prática clínica. Isto porque em muitos casos o paciente realmente necessita da polimedicação, sendo inviável a retirada de algum medicamento. Por exemplo, a associação de carbonato de lítio com antidepressivos foi relatada pelo estudo de Rosa et al. (2006) como extremamente benéfica no tratamento de pacientes bipolares com sintomas depressivos, justamente devido ao sinergismo que ocorre entre os fármacos, promovendo maior liberação de serotonina e/ou noradrenalina. Outro exemplo é a associação com benzodiazepínicos, os quais ajudam a controlar a agitação que pode ser um problema bastante comum em pacientes dependentes químicos devido às crises de abstinência. (ROSA et al., 2006).

O grande número de prescrições observadas por paciente (considerando que para todos os pacientes foi analisado um período de três semanas) denota também a grande dificuldade que o médico psiquiatra tem em ajustar a terapia para cada indivíduo, pois cada um apresenta necessidades específicas e reage de formas diferentes frente à administração de cada medicamento. Neste contexto é possível inferir que uma maior participação do profissional farmacêutico na construção da farmacoterapia pode ser extremamente benéfica, pois além de agregar conhecimentos técnicos ele pode acompanhar mais de perto os sinais, sintomas e necessidades do paciente internado e relatar ao médico quaisquer eventos relacionados à medicação.

Ide et al. (2009) reportam um caso em que farmacêuticos acompanharam uma hospitalização decorrente de intoxicação por lítio. Ao analisar os medicamentos tomados pelo paciente foi descoberta a interação que causou o internamento e os profissionais farmacêuticos conversaram com a equipe médica para que medidas fossem tomadas para controlar a intoxicação. Concluíram em seu estudo que a intervenção farmacêutica foi essencial no caso apresentado para que a melhora do paciente fosse a mais rápida possível, melhorando assim, a qualidade de vida do mesmo, pois consequências mais graves da intoxicação foram evitadas. Além disso, afirmam que analisar regularmente a medicação dos pacientes contribui para aperfeiçoar o cuidado médico na hospitalização. 


\section{CONCLUSÃO}

Na análise das 41 prescrições, foi possível observar cinco contendo carbonato de lítio associado a outros medicamentos e a presença de 19 interações. Seis interações foram classificadas como grave, onze moderadas e duas leves. A conduta clínica recomendada depende do tipo de interação e do medicamento utilizado e pode ser o monitoramento da ocorrência de reações adversas, observação de sintomas de intoxicação, ajuste de doses, suspensão da medicação ou nenhuma conduta. Diante desta situação, pode-se concluir que apesar da imperiosa necessidade de associações medicamentosas nas diferentes situações clínicas, é imprescindível o monitoramento do paciente para que a farmacoterapia seja considerada segura e eficaz. Fica evidente a necessidade de um maior cuidado e atenção entre os profissionais que prestam assistência aos pacientes internados, para que possam atuar prontamente e/ou profilaticamente quando houver sinais ou risco de toxicidade resultante das interações com carbonato de lítio. Nesta perspectiva, farmacêutico está inserido prestando cuidado ao paciente, de forma a otimizar a farmacoterapia, promover saúde e bem-estar e prevenir doenças.

\section{AGRADECIMENTOS}

A direção e ao serviço de Farmácia da Clínica de reabilitação da Clínica Quinta do Sol em Curitiba Pr.

\section{REFERÊNCIAS}

BOORA, K.; XU, J.; HYATT, J. Encephalopathy with combined lithium-risperidone administration. Acta Psychiatrica Scandinavica, v. 117, n. 5, 2008. Disponível em: < http://www.ncbi.nlm.nih.gov/pubmed/18331580>. Acesso em: 25/09/2016.

CORRER, J. C.; OTUKI, M. F. A Prática Farmacêutica na Farmácia Comunitária. Porto Alegre: Artmed, 2013. 440p.

DRUGS.COM. Drug Interaction Report. Disponível em: < https://www.drugs.com/drug_Interactions.php>. Acesso em: 24/09/2016.

DYNAMED. Lithium. Disponível em: http://portalsaude.saude.gov.br/index.php/o-ministerio/ 
principal/periodicos. Acesso em: 24/09/2016.

GOLDBERG, J. F. Treatment Guidelines: Current and Future Management of Bipolar Disorder. Journal of Clinical Psychiatry, v. 61, supp 13, p. 12-18, 2000.

GOODWIN, F.K.; ZIS, A.P. Lithium in the Treatment of Mania: Comparisons with Neuroleptics. Archives of General Psychiatry, v. 36, n. 8, p. 840-844, 1979.

HANEMANN, F.D.M. Carbonato de Lítio. Revista da Graduação. Editora Universitária da PUC-RS, v.3, n.1, Rio Grande do Sul, 2010.

HEALY, D. Mania: A Short History of Bipolar Disorder. Baltimore: The Johns Hopkins University Press, 2008.

IDE, N.; GOTOU, S.; MORI, M. Hospital pharmacists prevented advance of lithium intoxication through pharmaceutical interventions. Yakugaku Zasshi, v. 129, n. 8, p. 10071011, 2009.

KETTER, T.A. Monotherapy versus combined treatment with second-generation antipsychotics in bipolar disorder. Journal of Clinical Psychiatry, v. 69, sup. 5, p. 9-15, 2008.

MEDSCAPE. Drug interaction Checker. Disponível em: < http://reference.medscape.com/druginteractionchecker >. Acesso em: 24/09/2016.

OLIVEIRA JUNIOR, N. A.; ANDRÉA, E. M.; MACIEL, W. A.; SIQUEIRA, L. R.; ATIÉ, J.; COSENZA, R. O Eletrocardiograma e a Síndrome de QT Longo. Revista da SOCERJ. v. 17, n. 3, p. 177-182, 2004.

PULCHERIO, G.; VERNETTI, C.; STREY, M.N.; FALLER, S. Transtorno de estresse póstraumático em dependente do álcool. Revista de Psiquiatria Clínica, São Paulo, v. 35, n. 4, p. 154-158, 2008.

RANG, H.P.; DALE, M.M.; RITTER, J.M.; FLOWER, R.J.; HENDERSON, G. (Ed) Rang e Dale: Farmacologia. 7a ed. Rio de Janeiro: Elsevier, 2013. 
RIBEIRO, M.; LARANJEIRA, R.; CIVIDANES, G. Transtorno bipolar do humor e uso indevido de substâncias psicoativas. Revista de Psiquiatria Clínica, v.32, supl 1, p. 78-88, 2005.

ROSA, A.R.; KAPCZINSKI, F.; OLIVA, R.; STEIN, A.; BARROS, H.M.T.. Monitoramento da Adesão ao Tratamento com Lítio. Revista de Psiquiatria Clínica. São Paulo, v. 33, n. 5, p. 249-261,2006.

TISSINI, R. M. J. Intoxicação por Lítio. In: ANDRADE FILHO, A.; CAMPOLINA, D.; DIAS, M. B. Toxicologia na Prática Clínica. 2ª edição. Belo Horizonte: Folium, 2013. 700p.

VACHERON-TRYSTRAM, M.N.; BRAITMAN, A.; CHEREF, S.; AUFFRAY, L. Antipsychotics in bipolar disorders. L’Encéphale, v. 30, n. 5, p. 417-424, 2004.

VIEL, A. M.; PAES, J. T. R.; STESSUK, T.; DOS SANTOS, L. Interações medicamentosas potenciais com benzodiazepínicos em prescrições médicas de pacientes hospitalizados. Revista de Ciências Farmacêuticas Básica e Aplicada., v. 35, n. 4, p. 589-596, 2014. 